\title{
The Internal Friction and the Relaxation Time Spectrum of Ferroelectric Ceramic PZT Type
}

\author{
J. BARTKOWSKA AND J. ILCZUK* \\ Department of Materials Science, Faculty of Computer Science and Material Science \\ Silesian University, Żeromskiego 3, 41-200 Sosnowiec, Poland \\ The fractional Zener model with two spring-pots is proposed to de- \\ scription of relaxation time spectrum of ferroelectric ceramic material. This \\ model is based on fractional calculus. The influence of values of $a$ and $b$ \\ parameters on the shape of the relaxation time spectrum was investigated. \\ PACS numbers: 77.84.Dy, 72.15.Lh, 77.90.+k, 62.40.+i
}

\section{Introduction}

In recent years, there has been a considerable amount of works carried out on modelling the viscoelastic response of materials $[1,2]$ over range of temperature $(T)$ and time $(t)$ of frequency $(f)$. These investigations deal primary with the modelling of various viscoelastic functions. These functions can be described by the generalised Maxwell or the generalised Kelvin-Voigt models [1, 2]. Using these models to fit the data obtained in relaxation experiments requires the determination of number of fitting parameters.

An alternative approach has been used to model the viscoelastic behaviour of material that is based on the concept of the fractional differential and integral method. The method uses the idea that the spring and dashpot elements in the phenomenological models are replaced by spring-pot element.

Linear viscoelastic models are often presented as spectral models. Fractional models are amenable to analysis using the Fourier and Laplace transforms and they are described by the fractional derivative of $a$ order with respect to time with evidently $0<a \leq 1$.

Although direct measurement of the relaxation time spectrum is impossible, the main advantage of this modelling is to ensure consistency with linear viscoelastic theory.

In this paper we considered two fractional viscoelastic Zener models. One of these consisted of one spring-pot and two springs and second one consisted of two spring-pots and two springs. We apply the fractional Zener model with two spring-

*corresponding author; e-mail: jilczuk@us.edu.pl 
pots to description of relaxation time spectrum of ceramic material PZT type. PZT that is lead zirconate titanate $\mathrm{Pb}(\mathrm{Zr}, \mathrm{Ti}) \mathrm{O}_{3}$ is a solid solution between zirconate and lead titanate. The material of our investigation was multicomponent ceramic material PZT type about composition: $\left(\mathrm{Pb}_{0.9} \mathrm{Ba}_{0.1}\right)\left(\mathrm{Zr}_{0.53} \mathrm{Ti}_{0.47}\right)+2 \% \mathrm{~mol} \mathrm{Nb}_{2} \mathrm{O}_{5}$.

\section{Reasons of the modelling viscoelastic response of material}

The internal friction $\left(Q^{-1}\right)$ in ceramic PZT type $\left(\left(\mathrm{Pb}_{0.9} \mathrm{Ba}_{0.1}\right)\left(\mathrm{Zr}_{0.53} \mathrm{Ti}_{0.47}\right)+\right.$ $2 \%$ mol $\mathrm{Nb}_{2} \mathrm{O}_{5}$ ) was measured as a function of temperature. There are three peaks $P_{1}, P_{2}$ and $P_{\mathrm{F}}$ on the temperature dependence of the internal friction (Fig. 1). The $P_{\mathrm{F}}$ peak always appears at the Curie temperature and it is controlled by the phase transition from the ferroelectric to the paraelectric phase.

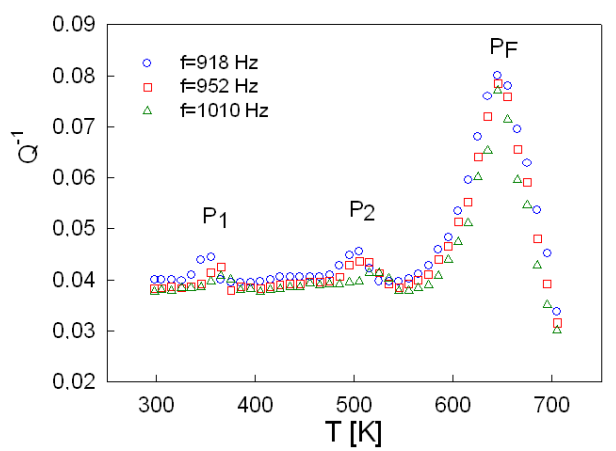

Fig. 1. The internal friction as function of temperature for three measurement frequency for the ceramic PZT type.

The $P_{1}$ and the $P_{2}$ peaks appear in the ferroelectric phase. The locations of $P_{1}$ and $P_{2}$ peaks depend on measurement frequency, thus their appearance on the temperature dependence of internal friction is controlled by relaxation processes. The subject of our investigations is description of mechanisms controlling the appearing of $P_{2}$ peak.

The first stage of investigation of mechanisms controlling appearance of the $P_{2}$ peak was describing this peak by the Wang model. There was good agreement between the Wang theory and experimental data. On the base of Wang's model we could affirm that the appearing of the $P_{2}$ peak, on the temperature dependence of the internal friction, is connected with viscoelastic domain walls mobility. This was presented in our last papers [3, 4].

The next stage of investigations of relaxation mechanisms, which control the appearing of $P_{2}$ peak, is working up the model of viscoelastic behaviour. The $P_{2}$ peak could be described by the model with more than one relaxation time like a parameter, because pre-exponential factor of the relaxation time, calculated from the law of Arrhenius, is very short $\left(\approx 10^{-19} \mathrm{~s}\right)$. 


\section{The fractional Zener model}

The models of material consist of the combination of free basic elements. The first element is a perfect elastic element (spring), the second one is perfect viscous element (dashpot) and the third one is viscoelastic element (spring-pot).

The Zener model is often used as a first approximation for the study of viscoelastic solids. The classical Zener model consists of the Maxwell body and a second spring parallel with it. The Maxwell model consists of one spring and one dashpot connected in series. If the dashpot is replaced by a spring-pot, then the Zener model becomes a fractional Zener model.

In this work, we adopted the constitutive equation introduced by Bagley and Torvik [2] to describe the viscoelastic behaviour of material. This equation, connecting the stress and the strain, for the fractional Zener model is following:

$$
\sigma(t)+\sum_{m=1}^{M} \tau_{m}^{a_{m}} D_{t}^{a_{m}} \sigma(t)=E_{0} \varepsilon(t)+\sum_{n=1}^{N} E_{n} \tau_{n}^{b_{n}} D_{t}^{b_{n}} \varepsilon(t),
$$

where $\sigma(t)$ and $\varepsilon(t)$ are the stress and strain respectively, $D_{t}^{a}(f)$ is the fractional derivative of $a$ or $b$ order of the $f$ function with respect to time with evidently $0<a \leq 1$ and $0<b \leq 1$. If parameter $a$ and $b$ is equal to 0 , viscoelastic element (spring-pot) becomes a perfect elastic element. The viscoelastic element becomes a perfect viscous element if $a$ and $b$ parameters are equal to 1 . If $m=n=1$, $a_{m}=b_{n}=a$, and $0<a \leq 1$, then Eq. (1) describes the fractional Zener model with one spring-pot.

\subsection{The fractional Zener model with one spring-pot}

The equation connecting the stress and the strain, for the fractional Zener model with one viscoelastic element is following:

$$
\sigma(t)+\tau^{a} D_{t}^{a} \sigma(t)=E_{0} \varepsilon(t)+E_{u} \tau^{a} D_{t}^{a} \varepsilon(t)
$$

where $\sigma(t)$ and $\varepsilon(t)$ are the stress and strain, respectively, $E_{0}$ and $E_{u}$ are relaxed and non-relaxed moduli and $\tau$ is the relaxation time. $D_{t}^{a}(f)$ is the fractional derivative of $a$ order of the $f$ function with respect to time with evidently $0<a \leq 1$.

After calculating the Fourier transform of the fractional Zener model with one spring-pot (Eq. (2)), we obtain the expression of the complex elastic modulus $E^{*}(\mathrm{i} \omega)$. The complex elastic modulus, real and imaginary parts of the complex modulus are following:

$$
\begin{aligned}
& E^{*}=\frac{E_{0}+E_{u}(\mathrm{i} \omega)^{a}}{1+(\mathrm{i} \omega \tau)^{a}}, \\
& E^{\prime}=E_{0} \frac{(1+k)(\omega \tau)^{2 a}+(\omega \tau)^{a}(2+k) \cos \left(a \frac{\pi}{2}\right)}{\left[1+(\omega \tau)^{a} \cos \left(a \frac{\pi}{2}\right)\right]^{2}+\left[1+(\omega \tau)^{a} \sin \left(a \frac{\pi}{2}\right)\right]^{2}}, \\
& E^{\prime \prime}=E_{0} \frac{k(\omega \tau)^{a}(2+k) \sin \left(a \frac{\pi}{2}\right)}{\left[1+(\omega \tau)^{a} \cos \left(a \frac{\pi}{2}\right)\right]^{2}+\left[1+(\omega \tau)^{a} \sin \left(a \frac{\pi}{2}\right)\right]^{2}},
\end{aligned}
$$

where $k=\frac{E_{u}-E_{0}}{E_{0}}$. The graph of dependence of the imaginary part on the real 
part of the complex elastic modulus, the so-called Cole-Cole diagram, was made on the base of Eqs. (4) and (5) (Fig. 2a). The Cole-Cole diagram was treated like the first test of the correctness of model assumptions.
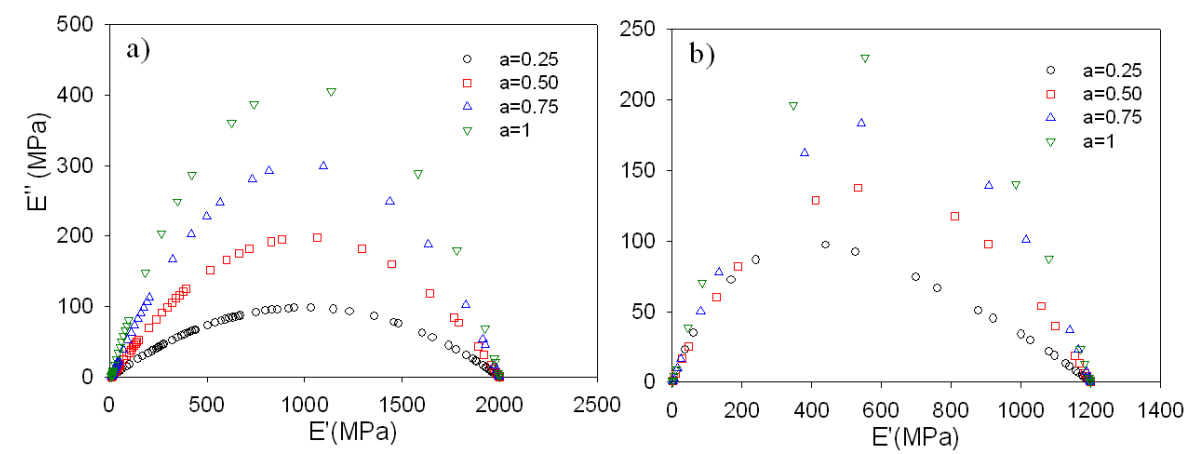

Fig. 2. Cole-Cole diagram for the fractional Zener model with one spring-pot (a), with two spring-pots (b).

The fractional Zener model with one spring-pot does not represent real viscoelastic behaviour of ceramic materials, because the Cole-Cole diagram for this model is symmetrical independently of $a$ parameter. The literature data show that the Cole-Cole diagram of the ceramic PZT type is asymmetrical $[5,6]$.

\subsection{The fractional Zener model with two spring-pots}

The fractional Zener model with two spring-pots consists of one spring and two spring-pots connected in series and second spring connected in parallel. This model is characterised by three mechanisms: the first one is associated with the viscoelastic behaviour at low temperature or at high frequency, the second one represents the viscoelastic behaviour at high temperature or at low frequency, the third one represents the elastic behaviour of the ceramic material.

After transformations analogous to calculations in the fractional Zener model with one spring-pot we received the expression of the complex elastic modulus $E^{*}(\mathrm{i} \omega)$ and the real and the imaginary parts of this complex modulus are the following, respectively:

$$
\begin{aligned}
& E^{*}=\frac{E_{u}-E_{0}\left[\left(\mathrm{i} s \tau_{0}\right)^{-a}\left(\mathrm{i} s \tau_{1}\right)^{-b}\right]}{1+\left(\mathrm{i} s \tau_{0}\right)^{-a}+\left(\mathrm{i} s \tau_{1}\right)^{-b}}, \\
& E^{\prime}=E_{0}=\frac{\left(E_{u}-E_{0}\right)(1+A)}{(1+A)^{2}+B^{2}}, \\
& E^{\prime \prime}=E_{0}=\frac{\left(E_{u}-E_{0}\right) B^{2}}{(1+A)^{2}+B^{2}},
\end{aligned}
$$

where

$$
A=\left(\frac{\tau_{0}}{\tau_{1}}\right)^{-b}\left(\omega \tau_{1}\right)^{-b} \cos \left(b \frac{\pi}{2}\right)+\left(\omega \tau_{1}\right)^{-a} \cos \left(a \frac{\pi}{2}\right),
$$




$$
B=\left(\frac{\tau_{0}}{\tau_{1}}\right)^{-b}\left(\omega \tau_{1}\right)^{-b} \sin \left(b \frac{\pi}{2}\right)+\left(\omega \tau_{1}\right)^{-a} \sin \left(a \frac{\pi}{2}\right) \text { and } 0<a \leq b \leq 1 .
$$

The graph of dependence of the real part on the imaginary part of the complex elastic modulus, the so-called Cole-Cole diagram, was made on the base of Eqs. from (7) to (10) (Fig. 2b).

The fractional Zener model with two spring-pots can represent real viscoelastic behaviour of ceramic materials of PZT type, because the Cole-Cole diagram for this model is asymmetrical for different values of $a$ parameter.

\section{The relaxation time spectrum}

In order to describe physical effects on the viscoelastic behaviour, the relaxation time spectrum is usually considered because function of relaxation time can be connected with process on a molecular level $[7,8]$.

Direct measurement of the relaxation time is impossible, therefore modelling of the relaxation time should be consistent with the linear viscoelastic theory. There are relations between the relaxation time and material functions like the relaxation modulus $E(t)$, the storage modulus $E^{\prime}(t)$ and the loss modulus $E^{\prime \prime}(t)$. These relations are specific kind of integral equations [9-11].

The solving of these specific kind of integral equations is complicated. The fractional calculus method approach was used to their solution [6-8].

We can obtain the expression of the relaxation time spectrum out of the special fractional calculus method [7-9] and of applying of the inverse Stieltjes transform [1]. The relaxation time spectrum can be calculated from the following equation:

$$
H(\tau)=\frac{E_{u}-E_{0}}{\pi} \frac{B^{2}}{(1+A)^{2}+B^{2}},
$$

where

$$
\begin{aligned}
& A=\left(\frac{\tau_{0}}{\tau_{1}}\right)^{a}\left(\omega \tau_{1}\right)^{-b} \cos (a \pi)+\left(\frac{\tau_{0}}{\tau_{1}}\right)^{a} \cos (b \pi), \\
& B=\left(\frac{\tau_{0}}{\tau_{1}}\right)^{a}\left(\omega \tau_{1}\right)^{-b} \sin (a \pi)+\left(\frac{\tau_{0}}{\tau_{1}}\right)^{a} \sin (b \pi), \quad \text { and } \quad 0<a \leq b \leq 1 .
\end{aligned}
$$

The plots of relaxation time spectrum for various values of $a$ parameter, with $b$ parameter constant, are presented in Fig. 3a. The function of the relaxation time spectrum $H(\tau) / E_{u}-E_{0}$ corresponds to density and it is calculated for fractional Zener model with two spring-pots from Eqs. from (11) to (13), and is presented in Fig. 3b.

The normalised relaxation time spectrum becomes narrow with increasing values of $b$ parameter. 

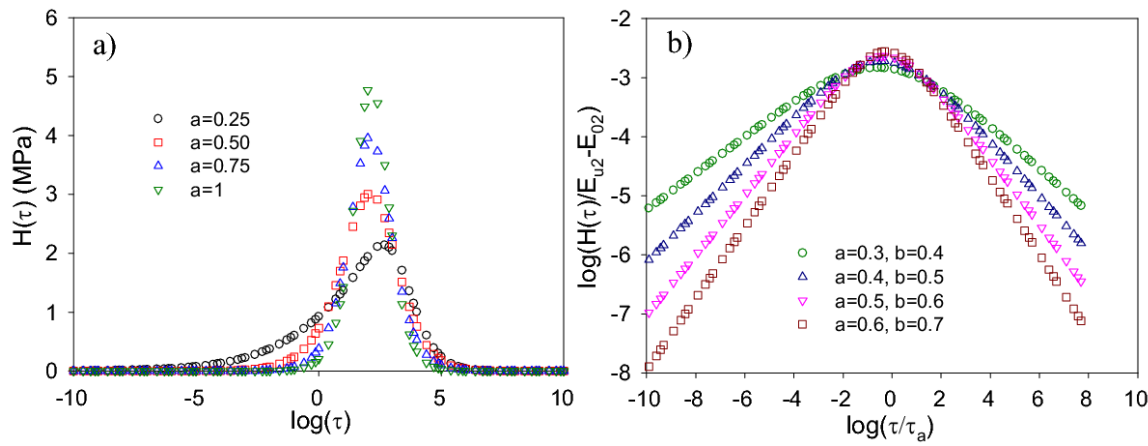

Fig. 3. Relaxation time spectrum calculated from the fractional Zener model with two spring-pots (a), logarithm of the normalised relaxation spectrum versus the normalised time for various values of $a$ and $b$ parameters (b).

\section{Conclusion}

In order to describe physical effects on the viscoelastic behaviour, the relaxation time spectrum is usually considered because function of relaxation time can be connected with process on a molecular level [9].

Direct measurement of the relaxation time is impossible, therefore modelling of the relaxation time should be consistency with the linear viscoelastic theory. There are relations between the relaxation time and material functions like the relaxation modulus $E(t)$, the storage modulus $E^{\prime}(t)$ and the loss modulus $E^{\prime \prime}(t)$. These relations are specific kind of integral equations [10].

The solving of these specific kind of integral equations is complicated. The fractional calculus method approach was used to their solution [6-8].

Then, we can draw conclusions from the theoretical consider that viscoelastic behaviour of ceramic material can be described by the theoretical viscoelastic model of material.

The fractional Zener model with two viscoelastic elements (spring-pots) describes viscoelastic behaviour of ceramic materials better than fractional Zener model with one spring-pot because the Cole-Cole diagram calculated from the model with two spring-pots is asymmetrical, that is this model gives results consistent with literature data.

The relaxation time spectrum was calculated from fractional Zener model with two spring-pots depending on values of $a$ and $b$ parameters. The shape of relaxation time spectrum depends on values of these parameters. The $a$ parameter relates to the broadening of the relaxation spectrum at low timescales, the $b$ parameter relates to the broadening of the relaxation spectrum at high timescales. The normalised relaxation time spectrum becomes narrow with increasing values of $b$ parameter if $(b-a)$ is constant.

Additionally the relaxation time spectra give a picture on the molecular mobility. 


\section{References}

[1] N.W. Tschoegl, The Phenomenological Theory of Linear Viscoelastic Behaviour. An Introduction, Springer-Verlag, New York 1988.

[2] R.L. Bagley, P.J. Torvik, J. Rheol. 27, 201 (1983).

[3] J.A. Bartkowska, J. Bluszcz, R. Zachariasz, J. Ilczuk, B. Brus, J. Phys. IV (France) 137, 19 (2006).

[4] J.A. Bartkowska, R. Zachariasz, J. Ilczuk, Mol. Quantum Acoust. 27, 25 (2006).

[5] K. Prabakar, S.P. Mallikarjun Rao, J. Alloys Comp. 437, 302 (2007).

[6] G.B. Davis, M. Kohandel, S. Sivaloganathan, G. Tenti, Med. Eng. Phys. 28, 455 (2006).

[7] A. Chatterjee, J. Sound Vibrat. 284, 1239 (2005).

[8] N. Heymans, Sign. Process. 83, 2345 (2003).

[9] R.C. Gloeckle, T.F. Nonnenmacher, Macromolecules 24, 6426 (1991).

[10] M. Alcoutlabi, J.J. Martinez-Vega, J. Mater. Sci. 34, 2361 (1999).

[11] H. Hammamia, M. Arousb, M. Lagachea, A. Kallel, Composites: Part A 37, 1 (2006). 